\title{
Amaurose bilateral por menigoencefalite criptocócica: relato de caso
}

\author{
Bilateral amaurosis due to cryptococcus meningoencephalitis: case report
}

\author{
Ricardo Evangelista Marrocos de Aragão ${ }^{1}$ \\ Cristina Muccioli ${ }^{2}$ \\ Ieda Maria Alexandre Barrreira ${ }^{3}$ \\ Daniel Canamary Silveira Ribeiro ${ }^{4}$ \\ Cristiane Nobrega Gularte Timóteo ${ }^{5}$
}

\begin{tabular}{|l|}
\hline RESUMO \\
\hline Meningoencefalite criptocócica é infecção causada por um fungo deno- \\
minado Cryptococcus neoformans. Duas formas são conhecidas: varia- \\
ção gattii e neoformans. A infecção antes da puberdade é rara. Cerca de \\
metade dos pacientes apresentam algum estado imunossupressivo. O \\
papiledema está presente em um terço dos pacientes por ocasião do \\
diagnóstico. Relatamos um caso de meningoencefalite por criptococose \\
em paciente de oito anos de idade, sem relato de doenças prévias, que \\
evoluiu com amaurose bilateral. O diagnóstico foi confirmado por de- \\
tecção do C. neoformans, var. gattii. O paciente foi tratado com an- \\
fotericina B e dexametasona. Na literatura existem poucos relatos de \\
perda visual permanente após meningite por criptococose. A existência \\
de um protocolo para tratamento de pacientes com papiledema é um fator \\
determinante para evitar a perda visual.
\end{tabular}

Descritores: Criptococose; Cegueira/etiologia; Cegueira/quimioterapia; Meningoencefalite/diagnóstico; Meningite; Hipertensão intracraniana; Papiledema

\footnotetext{
Trabalho realizado no Serviço de Oftalmologia do Hospital Universitário Walter Cantídio da Universidade Federal do Ceará - UFC - Fortaleza (CE) - Brasil.

Doutor em Medicina e especialista em Retina e Vítreo pela Universidade de Regensburg - Alemanha; Preceptor de Retina e Vítreo da Residência Médica de Oftalmologia do Hospital Universitário Walter Cantídio da Universidade Federal do Ceará - UFC - Fortaleza (CE) Brasil.

Livre Docente, Professora Adjunta, Chefe do Setor de Úvea e AIDS do Departamento de Oftalmologia da Universidade Federal de São Paulo - UNIFESP - São Paulo (SP) - Brasil.

Especialista em Retina e Vítreo pela UNIFESP - São Paulo (SP) - Brasil.

${ }^{4}$ Residente do terceiro ano do Serviço de Oftalmologia do Hospital Universitário Walter Cantídio da UFC . Fortaleza (CE) - Brasil.

${ }^{5}$ Residente do terceiro ano do Serviço de Oftalmologia do Hospital Universitário Walter Cantídio da UFC Fortaleza (CE) - Brasil

Endereço para correspondência: Ricardo E. Marrocos de Aragão. Rua Osvaldo Cruz, 2.335 - Fortaleza (CE) CEP 60125-151

E-mail: ricardomarrocos@yahoo.com

Recebido para publicação em 17.09.2006

Última versão recebida em 11.06.2007

Aprovação em 08.10.2007
}

\section{INTRODUÇÃO}

A criptococose, também conhecida como torulose ou blastomicose européia, é uma micose sistêmica com evolução subaguda ou crônica, causada pelo fungo Cryptococcus neoformans ${ }^{(1)}$ que é um basidiomiceto que se apresenta na forma tecidual como levedura encapsulada em duas variedades fúngicas, com quatro sorotipos: variedade neoformans (sorotipos A e D) e var. gattii (sorotipos B e C). Comprometimento da imunidade celular é o principal fator predisponente para a infecção por C. neoformans havendo aumento importante dos casos após o advento da AIDS e utilização de drogas imunosupressoras (var. neoformans, fungo oportunista), mas pode ocorrer num hospedeiro normal (var. gattii, patógeno primário). Este fungo tem predileção pelo sistema nervoso central (SNC), causando meningoencefalite. Infecção criptocócica do SNC é incomum em crianças e algumas vezes de difícil diagnóstico ${ }^{(2)}$. Através da inalação, os propágulos leveduriformes atingem os pulmões, podem regredir ou progredir com disseminação hematogênica para o cérebro e meninges, eventualmente para outros órgãos. O primeiro caso de publicação por $C$. neoformans foi detectado há cerca de cem anos por Busse e Buschke na Alemanha ${ }^{(3)}$. Entretanto, a criptococose começou a receber atenção especial a partir da década de 60, com registros mais freqüentes de ocorrência devido ao emprego de agressivas terapias imunossupressoras no combate a neoplasias. Contudo, os casos realmente emergiram na década de 80 com a pandemia da AIDS. De fato, hoje a criptococose acomete até um terço dos 
pacientes com infecção pelo HIV e figura em quarto lugar dentre a causa mortis destes pacientes ${ }^{(3)}$. A criptococose pode também ocorrer em pacientes imunocompetentes levando à perda visual em até $52 \%$ dos sobreviventes e está associada com atrofia óptica após edema do disco óptico em até $60 \%$ dos $\operatorname{casos}^{(4)}$. Este artigo relata o caso de uma criança imunocompetente com meningite criptocócica que evoluiu com perda visual bilateral, apesar do tratamento.

\section{RELATO DO CASO}

Paciente do sexo masculino, 8 anos, procedente do interior do estado do Ceará, foi admitido no Hospital das Clínicas (Hospital Universitário Walter Cantídio) em outubro de 2004, com história de cefaléia frontal que se iniciou há um mês, acompanhada de vômitos, febre, adinamia intensa e perda de peso. Em sua história pregressa, referiu que morava em casa de tijolo há sete anos, sem condições adequadas de higiene, sanitário e tratamento para água. Criavam galinhas, cabras e vacas. Referiu contato com pombos na casa do tio. No exame físico, apresentava discreto edema periorbital e facial e hepatomegalia. Não foi realizado exame de fundo de olho na admissão. Exame microscópico do líquor revelou leveduras capsuladas sugestivas de Cryptococcus sp. Sorologia para HIV negativa. A tomografia computadorizada (TC) do crânio revelou pequena formação cística adjacente ao ventrículo lateral direito. O resultado da cultura do líquor revelou Cryptococcus neoformans var. gattii. No exame do fundo de olho foi visto edema do disco óptico. Na pesquisa do reflexo pupilar foi evidenciado defeito aferente bilateral. Paciente evoluiu clinicamente de estado geral regular a grave (comatoso), com vômitos, febre alta, rigidez de nuca, tetraparesia mais acentuada à esquerda. Foi tratado com anfotericina $\mathrm{B}$, dose acumulada total de $1088,2 \mathrm{mg}$. Outras drogas utilizadas no tratamento foram: manitol e dexametasona endovenosa. Sua última TC mostrou aumento dos ventrículos devido a hidrocefalia compensada. Os últimos pareceres da neurologia e oftalmologia evidenciaram midríase bilateral permanente não reativa à luz. Exame de fundoscopia evidenciou edema no olho direito e palidez do disco óptico no olho esquerdo (Figuras 1 e 2). Paciente evoluiu com amaurose bilateral. Recebeu alta hospitalar após 77 dias em uso de fluconazol.

\section{DISCUSS ÃO}

A criptococose tem sido descrita em pacientes de todas as idades. Contudo, ocorre com mais freqüência em adultos jovens e pessoas de meia idade, especialmente do sexo masculino $^{(5-6)}$. É incomum na $1^{\underline{a}}$ década de vida e 80 a $85 \%$ de todos os casos ocorrem entre 20 e 60 anos de idade ${ }^{(7)}$. Na literatura, o crescimento do número de casos de criptococose nos últimos anos, com o advento da AIDS, é um fato incontestável ${ }^{(2-3,8)}$. Criptococos tem sido tradicionalmente descrito como um patógeno oportunista em hospedeiro imunocomprometido $^{(1,7)}$. Entretanto, também existem alguns relatos em indivíduos saudáveis, como neste caso.

Em um estudo brasileiro retrospectivo ${ }^{(1)}$, onde foram analisados 104 casos de meningoencefalite criptocócica, em relação à apresentação clínica, a cefaléia foi o sintoma mais comum, seguido de febre e rigidez de nuca. Alterações visuais foram relatadas em 23 pacientes, caracterizadas por diminuição da acuidade visual, fotofobia e diplopia (por paralisia do nervo abducente). Em 4 pacientes foi verificado papiledema.

A forma inicial mais comum de apresentação é a cefaléia, associada a náuseas, vômitos, letargia, e perda de peso. Os sintomas menos comuns são distúrbios visuais, sinais cerebelares e afasia. Na literatura mundial, pouco se relata sobre a perda visual ${ }^{(8)}$. Em um estudo multicêntrico australiano, relatos de 26 pacientes infectados com C. neoformans var. gattii, papiledema foi encontrado em $33 \%$, mas nenhum deles tinha

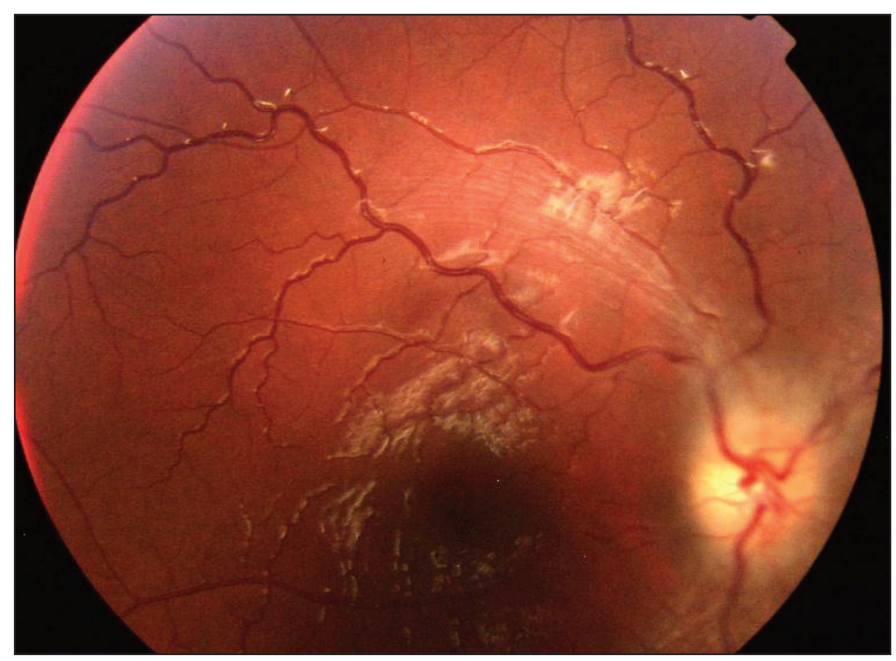

Figura 1 - Edema do disco óptico no olho direito

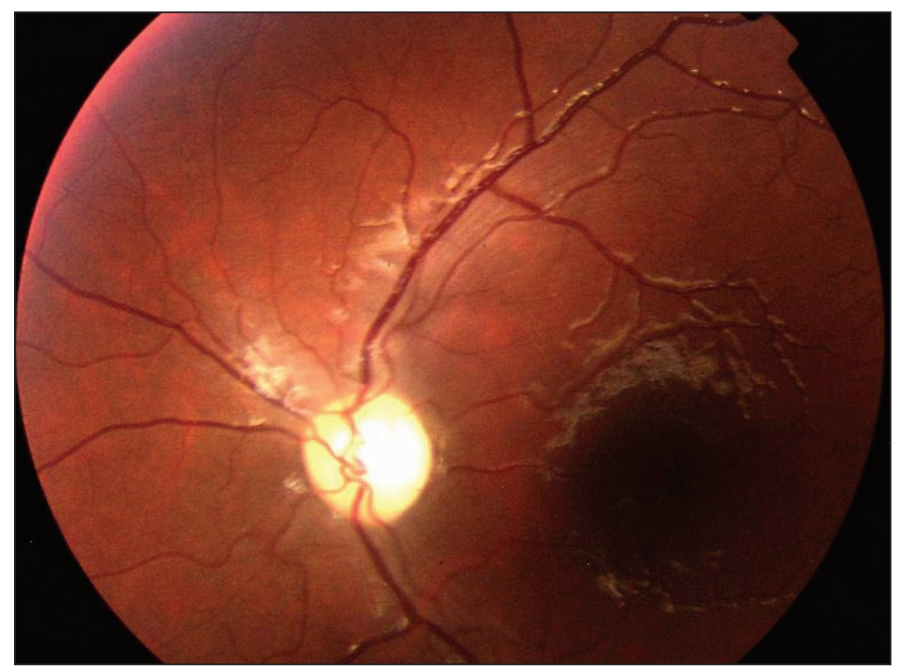

Figura 2 - Palidez do disco óptico no olho esquerdo 
relatado perda visual ${ }^{(4)}$. Em um estudo realizado em Papua Nova Guiné, a meningite criptocócica tem sido associada com uma considerável morbidade associada à perda visual. Os pacientes com hipertensão intracraniana foram tratados com procedimentos cirúrgicos.

Parece haver, algumas importantes diferenças com respeito ao risco de perda visual entre pacientes infectados com $C$. neoformans var. gattii, pois comparando-se pacientes imunocompetentes com imunossuprimidos observou-se que $52,5 \%$ dos pacientes sobreviventes imunocompetentes sofreram perdas visuais quando comparado a apenas $1,1 \%$ dos pacientes com AIDS. O padrão de perda visual lenta tem sido atribuído ao aumento da pressão intracraniana. No entanto, o aumento da pressão intracraniana e edema do disco óptico foram achados comuns, porém não associado à baixa de acuidade visual. Monitoramento da pressão liquórica intracraniana seria o ideal. Outro fator associado com a patogênese do edema do disco óptico, atrofia óptica e paralisia do nervo abducente é a alta titulagem de antígenos criptococos no líquor. Ainda neste estudo foi proposto que haveria um mecanismo de disfunção do nervo óptico relacionando o envolvimento da imunidade do paciente e não apenas a pressão liquórica.

Estudos histológicos das vias ópticas de pacientes com infecção por C. Neoformans var. gattii, demonstraram pequenas reações inflamatórias nas células, devido à invasão por criptococos. Isto leva à hipótese de pelo menos dois mecanismos que poderiam levar à disfunção do nervo óptico. Primeiramente, a resposta celular nas meninges cercando o nervo óptico pode causar um estrangulamento do nervo e comprometimento do seu suprimento vascular e como segundo mecanismo uma invasão direta do tecido neural pelo criptococos pode ser combinada com uma resposta do hospedeiro inflamatória celular causando edema no nervo óptico ${ }^{(4)}$. O que pode explicar os achados oftalmológicos encontrados no nosso caso clínico descrito.

Possíveis estratégias terapêuticas para se prevenir a perda visual, considera o controle da pressão liquórica, que incluem procedimentos de descompressão do nervo óptico. Porém, só esta medida pode não ser efetiva em pacientes imunocompetentes; nestes se sugere que o tratamento para redução da resposta inflamatória ao redor do nervo óptico pode ter potencial benéfico, com o uso de altas doses de corticosteróide sistêmico.

\section{ABSTRACT}

Cryptococcal meningitis is caused by the yeast Cryptococcus neoformans. Two varieties are recognized: var. gattii and var. neoformans. It is usually associated with immunosuppressive states, particularly HIV infection. Cryptococcal infection of the central nervous system is uncommon in immunocompetent children and difficult to diagnose. Ocular complications are common. Optic disc swelling was found in $33 \%$. The following report describes a case of meningitis caused by $C$. neoformans var. gattii in an 8 year-old immunocompetent child who developed optic atrophy. The patient was treated with amphotericin B associated with corticosteroids. Possible therapeutic strategies aimed at reducing visual loss in cryptococcal meningitis have great importance to avoid this important morbidity.

Keywords: Cryptococcosis; Blindness/etiology; Blindness/ drug therapy; Meningoencephalitis/diagnosis; Meningitis; Intracranial hypertension; Papilledema

\section{REFERÊNCIAS}

1. Darzé C, Lucena R, Gomes I, Melo A. Características clínicas laboratoriais de 104 casos de meningoencefalite criptocócica. Rev Soc Bras Med Trop. 2000;33(1):21-6.

2. Corrêa MPSC, Oliveira EC, Duarte RRBS, Pardal PPO, Oliveira FM, Severo LC. Criptococose em crianças no Estado do Pará, Brasil. Rev Soc Bras Med Trop. 1999;32(5):505-8.

3. Fortes AT, Assato MS, Fortes S, Lazera M. Meningoencefalite por Cryptococcus neoformans var gatti em indígena HIV negativo - relato de caso [texto na Internet]. In: 1 ${ }^{\text {er }}$ Encuentro Virtual de Neurocirugía 1999. [citado 2007 Jan 23] Disponível em: http://neuroc99.sld.cu/text/meningoencefalite.htm.

4. Seaton RA, Verma N, Naraqi S, Wembri JP, Warrell DA. Visual loss in immunocompetent patients with Cryptococcus neoformans var. gattii meningitis. Trans R Soc Trop Med Hyg. 1997;91(1):44-9. Comment in: Trans R Soc Trop Med Hyg. 1997;91(6):727-8.

5. Manfredi R, Nanetti A, Mazzoni A, Mastroianni A, Chiodo F. [The incidence, etiology and clinical significance of visceral mycoses in patients with AIDS]. Minerva Med. 1993;84(7-8):383-91. Italian.

6. Goodman JS, Koenig MG. Amphotericin B - Specifics of Administration. Mod Treat. 1970;7(3):581-95.

7. Hung PC, Wang HS, Chou ML, Sun PC, Huang SC. Cerebral cryptococcosis in a child. Zhonghua Min Guo Xiao Er Ke Yi Xue Hui Za Zhi. 1995; 36(2):131-5.

8. Williams TD. Diseases of the optic nerve, tracts, and visual cortex: An annual review of the ophthalmic literature. Am J Optom Physiol Opt. 1978; 55(9):652-9.

\section{Visite o site eletrônico dos ABO} www.aboonline.com.br 